\title{
Contribution to the study on chemical constituents from the leaves of Cassia alata I., (Caesalpiniaceae)
}

- Ngo Thi Thuy Duong

- Hoang Thi Chinh

- Thong Sui Din

- Truong Le Hung Phong

- Phan Nhat Phuong

- Pham Quoc Quynh

- Nguyen Tra Thanh Truc

- Ton That Quang

University of Science, VNU-HCM

(Manuscript received on March 20 2013, accepted on September $9^{\text {th }}$ 2013)

\section{ABSTRACT}

Six compounds were isolated from the leaves of Cassia alata L. (Caesalpiniaceae), including: aloe emodin (1), aloe emodin-8-O$\beta$-glucoside (2), rhein methyl ester (3), and phytol (6). Rhein methyl ester (3) was first isolated from the Cassia genus. Their chemical structures were elucidated by kaempferol (4), 4-hydroxybenzoic acid (5)

Key words: Cassia alata, anthraquinone.<smiles>CCOc1cccc2c1C(=O)c1cc(CO)cc(O)c1C2=O</smiles>

$\mathrm{R}=\mathrm{H}$<smiles>CC(=O)c1cc(O)c2c(c1)C(=O)c1cccc(O)c1C2=O</smiles>

Rhein methyl ester (3)<smiles>O=c1c(O)c(-c2ccc(O)cc2)oc2cccc(O)c12</smiles>

Kaempferol (4)<smiles>O=C(O)c1ccc(O)cc1</smiles>

4-Hydroxybenzoic acid (5)

Trang 26 


\section{INTRODUTION}

Cassia alata L. (= Senne alata L.) (Fig. 1) belongs to the Caesalpiniaceae family. It is native to Central America and has been introduced into many tropical countries and islands. In Vietnam, it is widely distributed in the Middle and the South [1, 2, 3, 4].

Cassia alata L. appears in a considerable number of published ethnopharmacological studies. It is laxative, antifebrile, antiseptic and diuretic. It has been used to cure many diseases: skin rashes, constipation, herpes circine, digestive ailments... [1, 2, 3, 4].

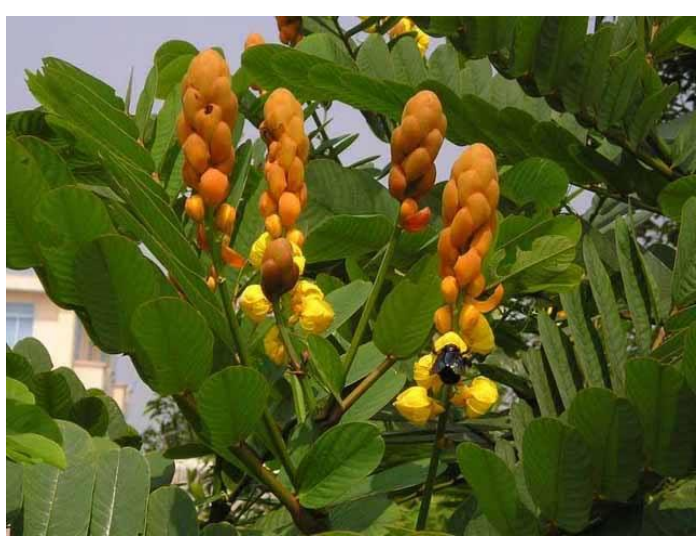

Fig. 1. Cassia alata L.

\section{MATERIALS AND METHODS}

\section{General}

NMR spectra were obtained on a Bruker Avance 500 NMR spectrometer. Mass spectra were taken on a high resolution ESI Bruker Daltonics micrOTOF-Q II 10187 mass spectrometer. Chromatography column was carried out on Kieselgel 60 (63-200 mesh) Merck. Thin-layer chromatography was performed on Merck 25DC-Alufolien $20 \times 20 \mathrm{~cm}$ Kieselgel 60 F254 plates. 1D and 2D-NMR were recorded on Bruker Avance 500 (500 $\mathrm{MHz}$ for ${ }^{1} \mathrm{H}-\mathrm{NMR}$ and $125 \mathrm{MHz}$ for $\left.{ }^{13} \mathrm{C}-\mathrm{NMR}\right)$.

\section{Plant material}

Plants were collected in Binh Chanh district, Ho Chi Minh City and was identified by pharmacist Phan Duc Binh, the deputy editor of the "Medicine and Health" semi-monthly magazine.

\section{Extraction and isolation}

Plants were washed, dried, ground into powder $(2.1 \mathrm{~kg})$ and exhaustedly extracted by methanol at room temperature. After evaporating, the methanolic solution gave crude extract (525 g). This extract was partitioned successively in petroleum ether, chloroform, ethyl acetate and butanol to give corresponding extracts. Petroleum ether fraction and ethyl acetate fraction were chromatographied repeatedly to afford six pure compounds. Petroleum ether fraction gave (3), (6) and ethyl acetate fraction gave (1), (2), (4), (5).

Aloe emodin (1): lightly orange needles. ${ }^{1} \mathrm{H}$ NMR (500 MHz, DMSO- $\left.d_{6}\right)$ : $11.96(2 \mathrm{H}$, br, $\mathrm{OH}), 7.82(1 \mathrm{H}, \mathrm{t}, J=8.0 \mathrm{~Hz}, \mathrm{H} 6), 7.74(1 \mathrm{H}, \mathrm{d}, J$ $=7.5 \mathrm{~Hz}, \mathrm{H} 5), 7.71(1 \mathrm{H}$, brs, H4), $7.40(1 \mathrm{H}, \mathrm{d}, J$ $=8.0 \mathrm{~Hz}, \mathrm{H} 7), 7.31(1 \mathrm{H}, \mathrm{brs}, \mathrm{H} 2), 5.62(2 \mathrm{H}, \mathrm{t}, J$ $\left.=6.0 \mathrm{~Hz}, 3-\mathrm{CH}_{2} \mathrm{OH}\right) .{ }^{13} \mathrm{C}-\mathrm{NMR}(125 \mathrm{MHz}$, DMSO- $d_{6}$ ): 161.6 (C1), 120.7 (C2), 153.7 (C3), 117.1 (C4), 119.3 (C5), 137.3 (C6), 124.4 (C7), 161.3 (C8), 191.6 (C9), 181.5 (C10), 133.4 (C11), 115.9 (C12), 114.5 (C13), 133.1 (C14), $62.0(\mathrm{C} 15)$.

Aloe emodin-8- $\boldsymbol{O}$ - $\boldsymbol{\beta}$-glucoside (2): orange needles. ${ }^{1} \mathrm{H}-\mathrm{NMR}$ (500 MHz, Pyridine- $d_{5}$ ): 13.34 (1H, br, $-\mathrm{OH}), 8.11$ (1H, brs, H4), 8.04 (1H, d, J $=8.0 \mathrm{~Hz}, \mathrm{H} 5), 7.99(1 \mathrm{H}, \mathrm{d}, J=8.0 \mathrm{~Hz}, \mathrm{H} 7), 7.67$ (1H, brs, H2), $7.60(1 \mathrm{H}, \mathrm{t}, J=8.0 \mathrm{~Hz}, \mathrm{H} 6), 5.00$ (3- $\left.\mathrm{CH}_{2} \mathrm{OH}\right), 5.81\left(1 \mathrm{H}, \mathrm{d}, J=7.5 \mathrm{~Hz}, \mathrm{H} 1^{\prime}\right.$ ), 4.60 $(1 \mathrm{H}, \mathrm{dd}, J=12.0 \mathrm{~Hz}, J=2.5 \mathrm{~Hz}, \mathrm{H6}$ 'a), 4.57 $\left(1 \mathrm{H}, \mathrm{t}, J=8.5 \mathrm{~Hz}, \mathrm{H} 2^{\prime}\right), 4.43(1 \mathrm{H}, \mathrm{t}, J=7.5 \mathrm{~Hz}$, H4'), $4.42(1 \mathrm{H}, \mathrm{dd}, J=12.5 \mathrm{~Hz}, \mathrm{~J}=5.5 \mathrm{~Hz}$, H6’b), 4.38 (1H, t, $J=8.5$ Hz, H3'), 4.25 (1H, m, H5'). ${ }^{13} \mathrm{C}-\mathrm{NMR}$ (125 MHz, Pyridine- $\left.d_{5}\right): 163.1$ (C1), 121.4 (C2), 153.2 (C3), 116.7 (C4), 121.2 (C5), 135.0 (C6), 123.0 (C7), 159.4 (C8), 188.6 (C9), 182.6 (C10), 133.1 (C11), 116.0 (C12), 
116.3 (C13), 127.0 (C14), 63.2 (C15), 102.7 (C1'), 74.9 (C2'), 78.4 (C3'), 71.1 (C4'), 79.4 (C5'), 62.4 (C6').

Rhein methyl ester (3): yellow powder. HRESI-MS analysis $\left(m / z 299.0587[\mathrm{M}+\mathrm{H}]^{+}\right.$, calcd. for $\left.\left[\mathrm{C}_{16} \mathrm{H}_{11} \mathrm{O}_{6}+\mathrm{H}\right]^{+}=299,0550\right) .{ }^{1} \mathrm{H}-\mathrm{NMR}(500$ $\left.\mathrm{MHz}, \mathrm{CDCl}_{3}\right): 12.03(1 \mathrm{H}, \mathrm{s}, 1-\mathrm{OH}), 11.97(1 \mathrm{H}, \mathrm{s}$, $8-\mathrm{OH}), 8.43(1 \mathrm{H}, \mathrm{d}, J=1.5 \mathrm{~Hz}, \mathrm{H} 4), 7.95(1 \mathrm{H}, \mathrm{d}$, $J=1.5 \mathrm{~Hz}, \mathrm{H} 2), 7.89(1 \mathrm{H}, \mathrm{dd}, J=7.5 \mathrm{~Hz}, J=1.0$ Hz, H5), 7.74 (1H, t, $J=8.0, \mathrm{H} 6), 7.37$ (1H, dd, $J$ $=8.5 \mathrm{~Hz}, J=1.0 \mathrm{~Hz}, \mathrm{H} 7), 4.00\left(3 \mathrm{H}, \mathrm{s},-\mathrm{OCH}_{3}\right)$. ${ }^{13} \mathrm{C}-\mathrm{NMR}\left(125 \mathrm{MHz}, \mathrm{CDCl}_{3}\right.$ ): 163.0 (C1), 125.5 (C2), 138.1 (C3), 120.4 (C4), 120.6 (C5), 137.9 (C6), 125.1 (C7), 162.6 (C8), 193.1 (C9), 181.1 (C10), 133.7 (C11), 116.1 (C12), 134.1 (C13), 118.5 (C14), 165.1 (C15), 53.0 (C16).

Kaempferol (4): yellow needles. ${ }^{1} \mathrm{H}-\mathrm{NMR}$ $\left(500 \mathrm{MHz}, \mathrm{DMSO}-d_{6}\right): 12.48(1 \mathrm{H}, \mathrm{s}, 5-\mathrm{OH})$ $10.79(1 \mathrm{H}, \mathrm{s}, 7-\mathrm{OH}), 10.11\left(1 \mathrm{H}, \mathrm{s}, 4^{\prime}-\mathrm{OH}\right), 9.39$ $(1 \mathrm{H}, \mathrm{s}, 3-\mathrm{OH}), 8.05\left(2 \mathrm{H}, \mathrm{d}, J=9.0 \mathrm{~Hz}, \mathrm{H} 2{ }^{\prime}\right.$ and H6'), 6.93 (2H, d, $J=9.0 \mathrm{~Hz}, \mathrm{H} 3$ ' and H5'), 6.44 $(1 \mathrm{H}, \mathrm{d}, J=2.0 \mathrm{~Hz}, \mathrm{H} 8), 6.20(1 \mathrm{H}, \mathrm{d}, J=2.0 \mathrm{~Hz}$, H6). ${ }^{13} \mathrm{C}-\mathrm{NMR}$ (125 MHz, DMSO- $\left.d_{6}\right): 146.8$ (C2), 135.7 (C3), 175.9 (C4), 160.7 (C5), 98.2 (C6), 163.9 (C7), 93.5 (C8), 156.2 (C9), 103.1 (C10), 121.7 (C1'), 129.5 (C2' and C6'), 135.7 (C3' and C5'), 159.2 (C4').

4-hydroxybenzoic acid (5): white needles. ${ }^{1} \mathrm{H}-\mathrm{NMR}\left(500 \mathrm{MHz}\right.$, acetone- $\left.d_{6}\right): 7.91(2 \mathrm{H}, \mathrm{d}, J=$ $8.5 \mathrm{~Hz}, \mathrm{H} 2$ and H6), $6.91(2 \mathrm{H}, \mathrm{d}, J=9.0 \mathrm{~Hz}, \mathrm{H} 3$ and H5. ${ }^{13} \mathrm{C}-\mathrm{NMR}\left(125 \mathrm{MHz}\right.$, acetone- $\left.d_{6}\right): 121.8$ (C1), 131.8 (C2, C6), 115.1 (C3, C5), 161.7 (C4), $166.7(-\mathrm{COOH})$

Phytol (6): white powder. ${ }^{1} \mathrm{H}-\mathrm{NMR}$ $\left(500 \mathrm{MHz}, \mathrm{CDCl}_{3}\right): 5.41(1 \mathrm{H}, \mathrm{t}, J=7.0, \mathrm{H} 2), 4.15$ $(2 \mathrm{H}, \mathrm{d}, J=6.5 \mathrm{~Hz}, \mathrm{H} 1$ ), $2.0-0.7$ (protons of $\mathrm{CH}$, $\left.\mathrm{CH}_{2}, \mathrm{CH}_{3}\right) .{ }^{13} \mathrm{C}-\mathrm{NMR}\left(125 \mathrm{MHz}, \mathrm{CDCl}_{3}\right): 59.61$ (C1), 123.34 (C2), 140.45 (C3), 40.04 (C4), 25.33 (C5), 36.86 (C6), 32.87 (C7), 37.55 (C8), 24.64 (C9), 37.62 (C10), 32.97 (C11), 37.48 (C12), 24.95 (C13), 39.56 (C14), 28.14 (C15), 22.85 (C16), 22.77 (C17).

\section{RESULTS AND DISCUSSION}

Compound (1) was obtained as lightly orange needles. The ${ }^{1} \mathrm{H}-\mathrm{NMR}$ (500MHz, DMSO$d_{6}$ ) spectrum showed the presence of five aromatic protons. In ring $\mathrm{A}$, there were three protons at $\delta 7.82(1 \mathrm{H}, \mathrm{t}, J=8.0 \mathrm{~Hz}, \mathrm{H} 6), 7.74$ $(1 \mathrm{H}, \mathrm{d}, J=7.5 \mathrm{~Hz}, \mathrm{H} 5)$ and $7.40(1 \mathrm{H}, \mathrm{d}, J=$ $8.0 \mathrm{~Hz}, \mathrm{H} 7)$. In ring $\mathrm{C}$, two protons broad singlet were resonated at $\delta 7.71(1 \mathrm{H}$, brs, $\mathrm{H} 4)$ and 7.31 $(1 \mathrm{H}$, brs, $\mathrm{H} 2)$. A low field signals at $\delta 11.96$ that integrated for two protons was attributed to two identical hydroxyl protons that were strongly chelated to a carbonyl group. A triplet at $\delta 5.62$, integrated for two protons, was suggested the presence of a hydoxymethyl group. The ${ }^{13} \mathrm{C}$ NMR spectrum showed the signals of 15 carbons, which included two carbonyl downfield signals at $\delta 191.6$ and 181.5, indicative the presence of the chelated and nonchelated carbonyl respectively. Based on its spectral data and by comparison with data from the literature, 1 was assigned as aloe emodin [5].

Compound (2) was isolated as orange needles. Although NMR spectra of 2 were run in pyridine- $d_{5}$ (different from the solvent DMSO- $d_{6}$ of 1 ), the structure of 2 was nearly similar to 1 with five aromatic protons: $8.04(1 \mathrm{H}, \mathrm{d}, J=$ $8.0 \mathrm{~Hz}, \mathrm{H} 5), 7.99$ (1H, d, $J=8.0 \mathrm{~Hz}, \mathrm{H} 7), 7.60$ $(1 \mathrm{H}, \mathrm{t}, J=8.0 \mathrm{~Hz}, \mathrm{H} 6)$ for ring A and $8.11(1 \mathrm{H}$, brs, H4), 7.67 (1H, brs, H2) for ring $\mathrm{C}$; two chelated and nonchelated carbonyl at $\delta 188.6$ and 182.6; one hydoxymethyl group $(\delta \mathrm{H} 5.00, \delta \mathrm{C}$ 63.2). The only different is that 2 has one chelated hydroxyl group and the second hydroxyl group was changed to $O$-glucoside. Based on HSQC and HMBC (table 1), this glucoside linked to $\mathrm{C} 8$ of the aglycon moiety. From these data, 2 was identified as aloe emodin-8- $O$ - $\beta$-glucoside.

Compound (3) was obtained as yellow powder. The HR-ESI-MS displayed the pseudomolecular ion peak at $\mathrm{m} / \mathrm{z} 299.0587$ $[\mathrm{M}+\mathrm{H}]^{+}$(calcd. for $\left[\mathrm{C}_{16} \mathrm{H}_{11} \mathrm{O}_{6}+\mathrm{H}\right]^{+}=299.0550$ ) 
corressponding to the molecular formula $\mathrm{C}_{16} \mathrm{H}_{11} \mathrm{O}_{6}$. The ${ }^{1} \mathrm{H}-\mathrm{NMR}$ spectrum supported the chelated nature of two hydroxyl groups to the carbonyl by the characteristic downfield signals at $\delta 12.03(1 \mathrm{H}, \mathrm{s}, 1-\mathrm{OH})$ and $11.97(1 \mathrm{H}, \mathrm{s}, 8$ $\mathrm{OH})$. A monosubstituted ring A was indicated by the three coupled protons at $\delta 7.89(1 \mathrm{H}, \mathrm{dd}, J=$ $7.5 \mathrm{~Hz}, J=1.0 \mathrm{~Hz}, \mathrm{H} 5), 7.74(1 \mathrm{H}, \mathrm{t}, J=8.0, \mathrm{H} 6)$ and $7.37(1 \mathrm{H}, \mathrm{dd}, J=8.5 \mathrm{~Hz}, J=1.0 \mathrm{~Hz}, \mathrm{H} 7)$. A disubstituted ring $\mathrm{C}$ was indicated by the two coupled protons at $\delta 8.43(1 \mathrm{H}, \mathrm{d}, J=1.5 \mathrm{~Hz}, \mathrm{H} 4)$; $7.95(1 \mathrm{H}, \mathrm{d}, J=1,5 \mathrm{~Hz}, \mathrm{H} 2)$.

Table 1. The ${ }^{1} \mathrm{H}-\mathrm{NMR}(500 \mathrm{MHz})$ and ${ }^{13} \mathrm{C}-\mathrm{NMR}(125 \mathrm{MHz})$ spectra for 1 and 2

\begin{tabular}{|c|c|c|c|c|c|c|}
\hline \multirow{3}{*}{$\mathrm{N}^{\mathrm{o}}$} & \multicolumn{2}{|c|}{ Compound (1) (DMSO- $\left.d_{6}\right)$} & \multicolumn{4}{|c|}{ Compound (2) (pyridine- $d_{5}$ ) } \\
\hline & \multirow[b]{2}{*}{$\delta_{\mathrm{C}}$} & \multirow[b]{2}{*}{$\delta_{\mathrm{H}}(J$, in $\mathrm{Hz})$} & \multicolumn{2}{|r|}{ HSQC } & \multirow{2}{*}{$\begin{array}{l}\text { HMBC } \\
\left({ }^{1} \mathrm{H} \rightarrow{ }^{13} \mathrm{C}\right)\end{array}$} & \multirow{2}{*}{$\begin{array}{l}\text { COSY } \\
\left({ }^{1} \mathrm{H} \leftrightarrow{ }^{1} \mathrm{H}\right)\end{array}$} \\
\hline & & & $\delta_{\mathrm{C}}$ & $\delta_{\mathrm{H}}(J$, in $\mathrm{Hz})$ & & \\
\hline 1 & 161.6 & & 163.1 & & & \\
\hline 2 & 120.7 & $7.31 \mathrm{brs}$ & 121.4 & $7.67 \mathrm{brs}$ & 4,13 & 4 \\
\hline 3 & 153.7 & & 153.2 & & & \\
\hline 4 & 117.1 & 7.71 brs & 116.7 & 8.11 brs & $2,10,13$ & 2 \\
\hline 5 & 119.3 & $7.74 \mathrm{~d}(7.5)$ & 121.2 & $8.04 \mathrm{~d}(8.0)$ & 10,12 & 6,7 \\
\hline 6 & 137.3 & $7.82 \mathrm{t}(8.0)$ & 135.0 & $7.60 \mathrm{t}(8.0)$ & 7,8 & 5,7 \\
\hline 7 & 124.4 & $7.40 \mathrm{~d}(8.0)$ & 123.0 & $7.99 \mathrm{~d}(8.0)$ & 12 & 6,5 \\
\hline 8 & 161.3 & & 159.4 & & & \\
\hline 9 & 191.6 & & 188.6 & & & \\
\hline 10 & 181.5 & & 182.6 & & & \\
\hline 11 & 133.4 & & 133.1 & & & \\
\hline 12 & 115.9 & & 116.0 & & & \\
\hline 13 & 114.5 & & 116.3 & & & \\
\hline 14 & 133.1 & & 127.0 & & & \\
\hline 15 & 62.0 & $5.62 \mathrm{t}(6.0)$ & 63.2 & 5.00 overlapped & $2,3,4$ & \\
\hline $1-\mathrm{OH}$ & & $11.96 \mathrm{br}$ & - & $13.34 \mathrm{br}$ & & \\
\hline $8-\mathrm{OH}$ & & $11.96 \mathrm{br}$ & - & & & \\
\hline $1^{\prime}$ & - & - & 102.7 & $5.81 \mathrm{~d}(7.5)$ & 8 & 2 ' \\
\hline $2^{\prime}$ & - & - & 74.9 & $4.57 \mathrm{t}(8.5)$ & & 1', 3' \\
\hline $3^{\prime}$ & - & - & 78.4 & $4.38 \mathrm{t}(8.5)$ & & $2^{\prime}, 4^{\prime}$ \\
\hline $4^{\prime}$ & - & - & 71.1 & $4.43 \mathrm{t}(7.5)$ & & $3^{\prime}, 5^{\prime}$ \\
\hline $5^{\prime}$ & - & - & 79.4 & $4.25 \mathrm{~m}$ & & 4', 6'a, 6’b \\
\hline $\begin{array}{l}6^{\prime} \mathrm{a} \\
6^{\prime} \mathrm{b}\end{array}$ & - & - & 62.4 & $\begin{array}{l}4.60 \mathrm{dd}(12.0,2.5) \\
4.42 \mathrm{dd}(12.5,5.5)\end{array}$ & & $\begin{array}{l}5^{\prime}, 6 \text { 'b } \\
5^{\prime}, 6 \text { 'a }\end{array}$ \\
\hline
\end{tabular}

A three-proton singlet at $\delta 4,00$ indicated the presence of a methoxy group. The ${ }^{13} \mathrm{C} \mathrm{NMR}$ spectrum showed the signals of one methyl ester group $\left(-\mathrm{COOCH}_{3}\right)$ at $\delta 165,1$ and 53,0 ; two chelated and nonchelated carbonyl carbons at $\delta$ 193,1 (C9) and 181,1 (C10); two oxygenated aromatic carbons at $\delta 163,0(\mathrm{C} 1)$ and 162,6 (C8). The NMR spectral data of compound (3) closely resembled the known compound rhein ${ }^{[6]}$ except the signals of one $-\mathrm{OCH}_{3}$ moiety. Besides, the HMBC showed the correlation between the proton of this methoxyl group with carbonyl ester, so compound (3) was established as rhein methyl ester. This is the first time that rhein methyl ester (3) was isolated from the Cassia genus.

Comparison of ${ }^{1} \mathrm{H}-\mathrm{NMR}$ and ${ }^{13} \mathrm{C}-\mathrm{NMR}$ data and physical data with those reported in the literature confirmed that compounds 4 - 6 were kaempferol (4) [7], 4-hydroxybenzoic acid (5) and phytol (6) [8]. 
Table 2. The ${ }^{1} \mathrm{H}-\mathrm{NMR}(500 \mathrm{MHz})$ and ${ }^{13} \mathrm{C}-\mathrm{NMR}(125 \mathrm{MHz})$ spectra for 3

\begin{tabular}{|c|c|c|c|}
\hline \multirow{3}{*}{$\mathrm{N}^{\mathrm{o}}$} & \multicolumn{3}{|c|}{ Compound $(\mathbf{3})\left(\mathrm{CDCl}_{3}\right)$} \\
\hline & \multicolumn{2}{|r|}{ HSQC } & \multirow{2}{*}{$\begin{array}{l}\mathrm{HMBC} \\
\left({ }^{1} \mathrm{H} \rightarrow{ }^{13} \mathrm{C}\right) \\
\end{array}$} \\
\hline & $\delta_{\mathrm{C}}$ & $\delta_{\mathrm{H}}(J$, in $\mathrm{Hz})$ & \\
\hline 1 & 163.0 & & \\
\hline 2 & 125.5 & $7.95 \mathrm{~d}(1.5)$ & $1,4,15$ \\
\hline 3 & 138.1 & & \\
\hline 4 & 120.4 & $8.43 \mathrm{~d}(1.5)$ & $2,10,14,15$ \\
\hline 5 & 120.6 & $7.89 \mathrm{dd}(7.5,1.0)$ & $7,10,12$ \\
\hline 6 & 137.9 & $7.74 \mathrm{t} \mathrm{(8.0)}$ & 8,11 \\
\hline 7 & 125.1 & $7.37 \mathrm{dd}(8.5,1.0)$ & $5,8,12$ \\
\hline 8 & 162.6 & & \\
\hline 9 & 193.1 & & \\
\hline 10 & 181.1 & & \\
\hline 11 & 133.7 & & \\
\hline 12 & 116.1 & & \\
\hline 13 & 134.1 & & \\
\hline 14 & 118.5 & & \\
\hline 15 & 165.1 & & \\
\hline 16 & 53.0 & $4.00 \mathrm{~s}$ & 15 \\
\hline $1-\mathrm{OH}$ & - & $12.03 \mathrm{~s}$ & 1,2 \\
\hline $8-\mathrm{OH}$ & - & $11.97 \mathrm{~s}$ & $7,8,12$ \\
\hline
\end{tabular}

\section{Góp phần tìm hiểu thành phần hóa học lá cây Muồng Trâu Cassia alata I., (Caesalpiniaceae)}

- Ngô Thị Thùy Dương

- Hoàng Thị Chinh

- Thòng Sủi Din

- Trương Lê Hùng Phong

- Phan Nhật Phương

- Phạm Quốc Quỳnh

- Nguyễn Trà Thanh Trúc

- Tôn Thất Quang

Trường Đại học Khoa học Tự nhiên, ĐHQG-HCM 


\section{TÓM TÁT}

Từ lá cây Muồng Trâu Cassia alata L. (Caesalpiniaceae), đã cô lập được sáu hợp chất, bao gồm: aloe emodin (1), aloe emodin-8-O- $\beta$-glucosid (2), rhein metyl ester (3), kaempferol (4), acid 4-hydroxybenzoic

Từ khóa: Cassia alata, anthraquinone.

\section{REFERENCES}

[1] Võ Văn Chi, Từ điển cây thuốc Việt Nam, Nhà xuất bản Y Học (1997).

[2] Phạm Hoàng Hộ, Cây cỏ Việt Nam, Nhà xuất bản Trẻ (2000).

[3] Đỗ Tất Lợi, Những cây thuốc và vị thuốc Việt Nam, Nhà xuất bản Khoa học Kỹ thuật (1986).

[4] T. Hennebelle, B. Weniger, H. Joseph, S. Sahpaz, F. Bailleul, Senna alata, Fitoterapia, 385 - 393 (2009).

[5] R.M. Coopoosamy, M.L. Magwa, Antibacterial activity of aloe emodin and aloin A isolated from Aloe excelsa, African
(5) và phytol (6). Trong đó, hợp chất rhein methyl ester (3) lần đầu tiên được cô lập từ chi Cassia. Cấu trúc của các hợp chất được xác định bằng các phương pháp phổ nghiệm.
Journal of Biotechnology 5, 1092-1094 (2006).

[6] K. Danielsen, D.W. Aksnes, G.W. Francis, NMR study of some anthraquinones from rhubarb, Magn Reson Chem 30, 359-360 (1992).

[7] A.S. Hamzah, N.H. Lajis, Chemical constituents of Hedyotis herbacea, ASEAN Review of Biodiversity and Environmental Conservation 2, 1-5 (1998).

[8] T.T. Tong, J.Y. Chou-Hwang, An optically active chromanone from Gynura formosana, Phytochemistry 44, 553(1997). 